\title{
Cerebral blood flow and metabolism in patients with silent brain infarction: occult misery perfusion in the cerebral cortex
}

Hiroshi Nakane, Setsuro Ibayashi, Kenichiro Fujii, Seizo Sadoshima, Katsumi Irie, Takanari Kitazono, Masatoshi Fujishima

\begin{abstract}
Objectives-Silent brain infarction (SBI) is of growing interest as a possible risk factor for symptomatic stroke. Although morphological characteristics of SBI have been well defined, their characteristic patterns of cerebral blood flow (CBF) and metabolism are in dispute. The purpose of this study was to elucidate CBF and metabolism in patients with SBI in relation to symptomatic stroke.

Methods-The patients underwent PET and were separated into three groups; control group (C group), with no lesions on CT (n=9, mean age 57$)$, SBI group, with no neurological signs or history of stroke, but with ischaemic lesions on CT $(n=9$, mean age 63), and brain infarction group (BI group), with neurological deficits and compatible CT lesions in the area supplied by perforating arteries $(n=19$, mean age 56). Regional CBF, oxygen extraction fraction (OEF), cerebral metabolic rate for oxygen $\left(\mathrm{CMRO}_{2}\right)$, and cerebral blood volume (CBV) were measured by PET.
\end{abstract}

Results-Mean values for CBF to the cerebral cortex and deep grey matter were lower in the SBI group (31.6 (SD 5.8) and 34.3 (SD 6.9) $\mathrm{ml} / 100 \mathrm{~g} / \mathrm{min}$, respectively) and in the BI group (30.8 (SD 5.2), 33.9 (SD 5.9), respectively) than in the $C$ group (36.0 (SD 6.6) and 43.5 (SD 9.5), respectively). Although mean $\mathrm{CMRO}_{2}$ of deep grey matter (2.36 (SD 0.52) $\mathrm{ml} / 100 \mathrm{~g} / \mathrm{min}$ ) was significantly decreased in the SBI group compared with the $\mathrm{C}$ group (2.76 (SD 0.480), p<0.01), $\mathrm{CMRO}_{2}$ of the cortical area was as well preserved in the SBI patients (2.36 (SD 0.39)) as in the controls (2.48 (SD 0.32)) with a compensatory increase of mean OEF (0.45 (SD 0.06) and 0.41 (SD 0.05), respectively).

Conclusions-Patients with SBI showed decreased $\mathrm{CBF}$ and $\mathrm{CMRO}_{2}$ in deep grey matter. On the other hand, decreased CBF with milder increased OEF, resulting in preserved $\mathrm{CMRO}_{2}$ in the cerebral cortex indicates the presence of occult misery perfusion, suggesting that patients with SBI have reduced cerebral perfusional reserves.

\section{(F Neurol Neurosurg Psychiatry 1998;65:317-321)}

Keywords: cerebral infarction; positron emission tomography; cerebral blood flow
Recent advances in neuroimaging techniques have disclosed frequent silent brain infarctions (SBIs). Many reports have focused on the morphological characteristics of SBIs. Silent brain infarctions are usually located in the basal ganglia, ${ }^{1-4}$ and the size and number of SBIs are less than those of symptomatic brain infarctions. ${ }^{25}$ Aging, hypertension, and atrial fibrillation are closely related to SBIs. ${ }^{2-467}$ Moreover, nocturnal hypotension may play an important part in the development of SBIs. ${ }^{89}$

Whether SBIs affect cognitive function, ${ }^{510}$ recurrence of vascular disease, ${ }^{11}{ }^{12}$ residual handicap,,$^{51314}$ and mortality ${ }^{511}$ is still controversial. Thus it is important to evaluate changes in cerebral blood flow (CBF) and metabolism among patients with SBIs in comparison with those with symptomatic brain infarction or control patients. Only a few reports are available about $\mathrm{CBF}$ and metabolism in patients with SBIs and results are controversial. The purpose of this study was to investigate $\mathrm{CBF}$ and oxygen metabolism among patients with or without SBI, using PET.

\section{Materials and methods}

Two hundred and twenty nine patients were admitted to our clinic in Kyushu University hospital from 1986 to 1993 for PET to evaluate brain function. This study was approved by an institutional review committee and patients gave informed consent. We focused on patients who had symptomatic or asymptomatic lesions in the territory of perforating arteries because SBIs usually occur in this area. Patients who had cardiogenic embolism, atherothrombotic brain infarction, brain haemorrhage, dementia, metabolic encephalopathy, or other cerebral diseases were excluded. Patients with either occlusion or severe stenosis (more than 70\%) of the internal carotid or middle cerebral arteries were also excluded. We included patients who had transient global amnesia in this study. The cause of transient global amnesia remains unclear and several underlying conditions, such as transient ischaemic attacks, ${ }^{15}$ epilepsy, ${ }^{16}$ and migraine, ${ }^{17}$ are reported to be possible candidates. We previously reported that $\mathrm{CBF}$ and cerebral oxygen metabolism in patients with recent transient global amnesia were better preserved compared with those with transient ischaemic attacks. ${ }^{18}$ The patients with transient global amnesia seem to have less vascular disease and vascular risk factors than those with transient ischaemic attacks. ${ }^{19}$ Considering these findings together, we think that 
Table 1 Classification of patients (total of 229) examined by PET

\begin{tabular}{lrl}
\hline No symptoms and no lesions on CT & 9 & $\rightarrow$ Control group \\
Brain infarction (BI) & 122 & $\rightarrow$ SBI group \\
Asymptomatic & 9 & \\
Symptomatic & 94 & \\
$\quad$ Cardiogenic embolism & 5 & \\
Thrombosis & 80 & \\
ICA or MCA occlusion or severe stenosis: & 56 & \\
+ & 24 & \\
$-\quad$ & 5 & \\
Cortical lesion: & 19 & $\rightarrow$ BI group \\
$\quad+$ & 9 & \\
- & 20 & \\
Unclassified & 7 & \\
Transient ischaemic attack & 33 & \\
Brain haemorrhage & 5 & \\
Dementia & 18 & \\
Brain tumour & 10 & \\
Neurological degenerative disease & 24 & \\
Metabolic encephalopathy & & \\
Others &
\end{tabular}

ICA=internal carotid artery; $\mathrm{MCA}=$ middle cerebral artery.

cerebral ischaemia is not a major mechanism of transient global amnesia. Therefore, 37 patients were included in the present study. They were divided into three groups; control subjects, who had no neurological deficits, no episodes of stroke, and no lesions on CT (C group), patients with SBI, who had no neurological symptoms or history of stroke, but had silent CT infarcts (SBI group), and patients with symptomatic brain infarctions, who had neurological deficits and infarcts in the territory of the perforating arteries (BI group). There were nine patients in the $\mathrm{C}$ group (mean age 57 (9) years, MABP 114 (24 $\mathrm{mm} \mathrm{Hg}$ ), nine in the SBI group (mean age 64 (3 years, MABP 120 (20) $\mathrm{mm} \mathrm{Hg}$ ), and 19 in the BI group (mean age 56 (11) years, MABP 107 (17) $\mathrm{mm} \mathrm{Hg}$ ) in this study (table 1).
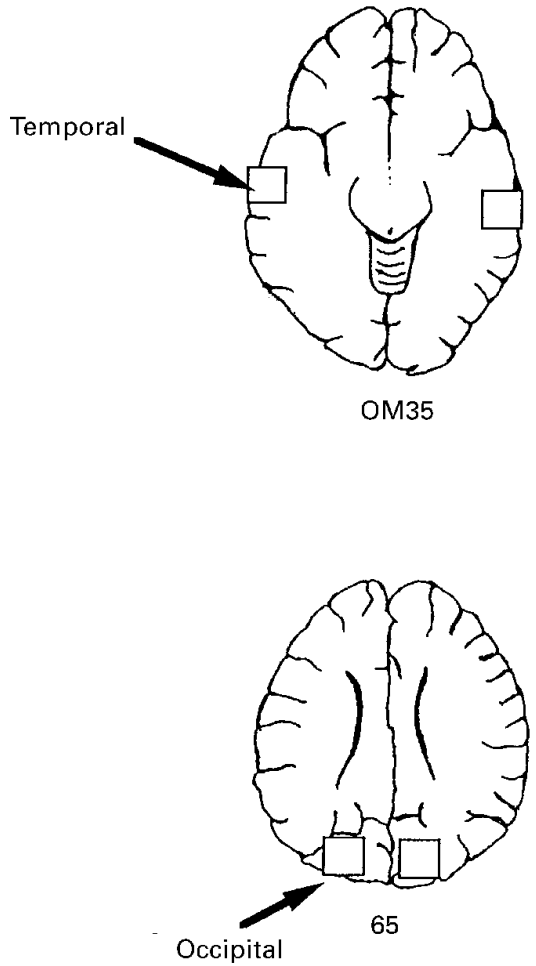

Figure 1 Schematic drawings showing locations of regions of interest (ROIs) placed symmetrically for each hemisphere. The numerals below each slice indicate distance from the orbitomeatal (OM) line in $\mathrm{mm}$.
Brain PET studies in chronic stages were performed within two months of admission. Regional (r) $\mathrm{CBF}$ and oxygen extraction fraction $(\mathrm{OEF})$ were measured by the $\mathrm{H}_{2}{ }^{15} \mathrm{O}$ continuous infusion method, according to the oxygen-15 steady state technique described by Frackowiak et al. ${ }^{20}$ Regional cerebral metabolic rates for oxygen $\left(\mathrm{CMRO}_{2}\right)$ were calculated as $\mathrm{rCMRO}_{2}=\mathrm{rCBF} \times \mathrm{rOEF} \times$ arterial oxygen content. Both rOEF and $\mathrm{rCMRO}_{2}$ were corrected using regional cerebral blood volume (rCBV) measured using a single inhalation of $\mathrm{C}^{15} \mathrm{O}_{2}$ gas. ${ }^{21}$ We excluded patients who had extremely low or high arterial $\mathrm{pCO}_{2}$ because of hyperventilation or respiratory disease. The HEADTOME-III device (Shimadzu Inc, Kyoto, Japan and Akita Noken, Akita, Japan) with a spatial resolution of $8.2 \mathrm{~mm}$ full width at half maximum was used as previously described. ${ }^{22}$ In each brain, the values in four slices parallel to the orbitomeatal (OM) line (35, 50, 65, and $80 \mathrm{~mm}$ above the OM line) were analysed. As shown in figure $1,18 \times 14 \mathrm{~mm}$ regions of interest (ROIs) were selected bilaterally in the frontal, temporal, parietal, and occipital cortices, striatum, and thalamus by the use of CT images corresponding to each PET slice. We used ROIs which did not include infarcted tissue. Values of all pixels in each ROI were averaged, and $\mathrm{rCBF}, \mathrm{rOEF}, \mathrm{rCMRO}_{2}$, and rCBV were determined. Mean values for the cerebral cortex were calculated by combining measurements in all cortical regions and for deep grey matter they were calculated by combining values in the striatum and thalamus. We also evaluated brain lesions on CT and
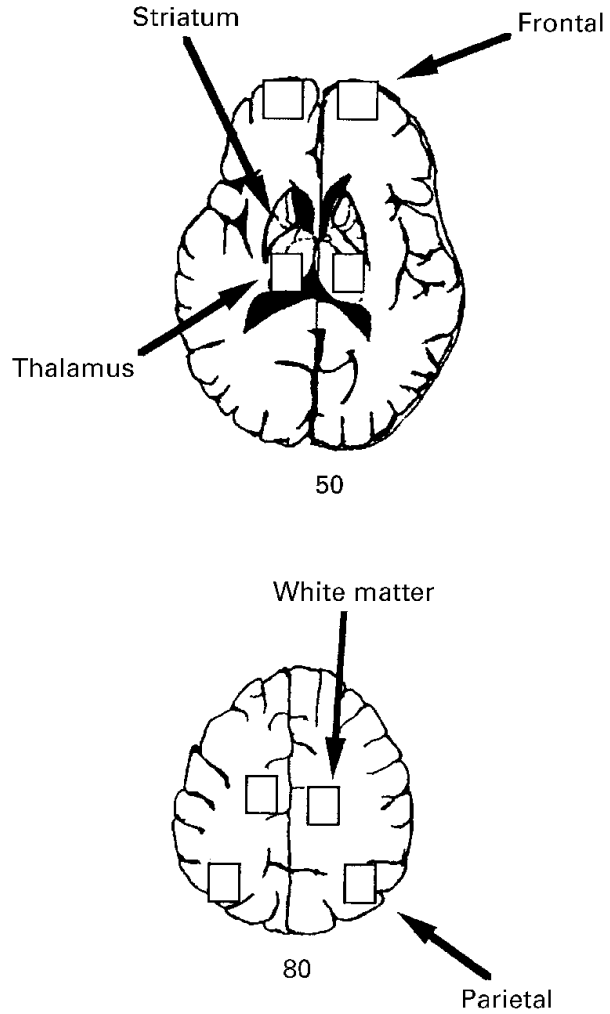
Table 2 Profiles of patients in control group and silent brain infarction (SBI) group

\begin{tabular}{lll}
\hline Cases & Age/sex & Diagnosis \\
\hline Control group: & & \\
1 & $53 / \mathrm{M}$ & HT \\
2 & $53 / \mathrm{M}$ & TGA \\
3 & $73 / \mathrm{F}$ & HT \\
4 & $65 / \mathrm{F}$ & HT \\
5 & $49 / \mathrm{F}$ & HT \\
6 & $47 / \mathrm{F}$ & HT \\
7 & $53 / \mathrm{F}$ & HT \\
8 & $58 / \mathrm{F}$ & HT \\
9 & $65 / \mathrm{F}$ & HT \\
SBI group: & & \\
1 & $66 / \mathrm{M}$ & DM \\
2 & $65 / \mathrm{M}$ & Otogenic vertigo \\
3 & $62 / \mathrm{F}$ & TGA \\
4 & $58 / \mathrm{M}$ & HT \\
5 & $65 / \mathrm{M}$ & TGA \\
6 & $67 / \mathrm{F}$ & TGA \\
7 & $64 / \mathrm{F}$ & HT \\
8 & $62 / \mathrm{M}$ & DM \\
9 & $62 / \mathrm{M}$ & Transient visual loss \\
& & \\
\hline
\end{tabular}

HT=hypertension; TGA=transient global amnesia; $\mathrm{DM}=$ diabetes mellitus.

counted the number of lesions in four CT slices parallel to the OM line.

We examined risk factors for cardiovascular disease, such as hypertension, diabetes mellitus (fasting blood glucose $>110 \mathrm{mg} / \mathrm{dl}$ ), hypercholesterolaemia (serum total cholesterol $>220$ $\mathrm{mg} / \mathrm{dl}$ ), and heart disease.

The values for $\mathrm{rCBF}$, rOEF, $\mathrm{rCMRO}_{2}$, $\mathrm{rCBV}$, and $\mathrm{rCBF} / \mathrm{rCBV}$ of each ROI were compared among the three groups. Mean values for the cerebral cortex and for deep grey matter for $\mathrm{CBF}, \mathrm{OEF}$, and $\mathrm{CMRO}_{2}$ were also compared among the three groups.

STATISTICS

We used the Kruskal-Wallis test to compare age, sex, and risk factors for cardiovascular disease among the three groups and a MannWhitney $U$ test to compare the number of symptomatic and asymptomatic lesions between BI and SBI groups. One way analysis of variance (ANOVA) followed by Scheffe's test was used to compare values of $\mathrm{rCBF}$, rOEF,

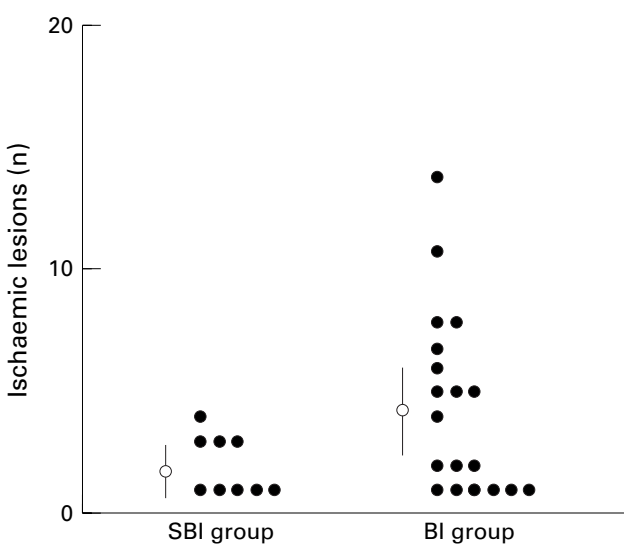

Figure 2 Number of ischaemic lesions in the brain, either symptomatic or asymptomatic in each patient in SBI and BI groups. Although there was no significant difference between groups, the BI group tended to have more lesions than the SBI groups. $S B I=$ silent brain infarction; $B I=$ brain infarction.

$\mathrm{rCMRO}_{2}, \mathrm{rCBV}$, and $\mathrm{rCBF} / \mathrm{rCBV}$ among the three groups. All values are presented as mean (SD).

\section{Results}

DEMOGRAPHICS OF PATIENTS

There were no significant differences in age among the three groups. The frequencies of risk factors for CVD (hypertension, diabetes mellitus, hypercholesterolaemia, and cardiac disease) were $88 \%, 0 \%, 33 \%$, and $22 \%$ respectively in the $\mathrm{C}$ group, $78 \%, 33 \%, 56 \%$, and $44 \%$ respectively in the SBI group, and $74 \%$, $32 \%, 42 \%$, and $11 \%$ respectively in the BI group. The number of patients with diabetes mellitus in the $\mathrm{C}$ group tended to be smaller than in the other groups, but there were no significant differences for risk factors among the groups (table 2).

Figure 2 shows the number of ischaemic lesions in the brain in each patient with SBI or BI. The SBI group had 2.0 (SD 1.2) lesions and the BI group had 4.5 (SD 3.7) lesions. The

Table 3 Regional cerebral blood flow, oxygen extraction fraction, cerebral metabolic rate for oxygen, and cerebral blood volume in the control, silent brain infarction (SBI), and brain infarction (BI) groups

\begin{tabular}{|c|c|c|c|c|c|c|}
\hline & \multicolumn{4}{|l|}{ Cerebral cortex } & \multicolumn{2}{|c|}{ Deep grey matter } \\
\hline & Frontal & Temporal & Parietal & Occipital & Striatum & Thalamus \\
\hline \multicolumn{7}{|c|}{$\mathrm{rCBF}(\mathrm{ml} / 100 \mathrm{~g} / \mathrm{min}):$} \\
\hline Control & $35.7(7.5)$ & $35.8(6.5)$ & $37.2(5.8)$ & $35.3(7.0)$ & $43.4(9.6)$ & $43.5(9.6)$ \\
\hline SBI & $31.3(6.4)$ & $30.8(5.1)^{\star}$ & $32.0(6.3)^{\star}$ & $32.3(5.0)$ & $36.5(7.6)^{\star}$ & $32.2(5.1)^{\star \star}$ \\
\hline BI & $29.6(4.7)^{\star \star}$ & $30.8(4.8)^{\star \star}$ & $31.9(5.5)^{\star \star}$ & $30.7(5.3)^{\star}$ & $35.6(5.8)^{\star \star}$ & $32.2(5.3)^{\star \star}$ \\
\hline \multicolumn{7}{|l|}{ rOEF: } \\
\hline Control & $0.40(0.04)$ & $0.40(0.04)$ & $0.42(0.05)$ & $0.44(0.05)$ & $0.41(0.05)$ & $0.36(0.04)$ \\
\hline SBI & $0.43(0.05)$ & $0.45(0.06)^{\star}$ & $0.43(0.05)$ & $0.47(0.06)$ & $0.43(0.07)$ & $0.39(0.06)$ \\
\hline $\mathrm{BI}$ & $0.43(0.05)$ & $0.43(0.05)$ & $0.44(0.05)$ & $0.45(0.05)$ & $0.42(0.05)$ & $0.36(0.05)$ \\
\hline \multicolumn{7}{|c|}{$\mathrm{rCMRO}_{2}(\mathrm{ml} / 100 \mathrm{~g} / \mathrm{min}):$} \\
\hline Control & $2.34(0.29)$ & $2.39(0.26)$ & $2.58(0.37)$ & $2.59(0.33)$ & $2.97(0.46)$ & $2.55(0.42)$ \\
\hline SBI & $2.22(0.31)$ & $2.31(0.32)$ & $2.32(0.36)$ & $2.57(0.46)$ & $2.62(0.57)$ & $2.10(0.26)^{\star \star}$ \\
\hline $\mathrm{BI}$ & $2.18(0.31)$ & $2.30(0.28)$ & $2.38(0.27)$ & $2.40(0.37)$ & $2.55(0.34)^{\star \star}$ & $1.99(0.39)^{\star \star}$ \\
\hline \multicolumn{7}{|c|}{$\mathrm{rCBV}(\mathrm{ml} / 100 \mathrm{~g}):$} \\
\hline Control & $3.38(0.62)$ & $3.68(0.81)$ & $3.11(0.47)$ & $3.82(1.52)$ & $3.06(0.55)$ & $3.80(1.03)$ \\
\hline SBI & $2.85(0.81)^{\star}$ & $3.00(0.65)^{\star \star}$ & $2.70(0.57)^{\star}$ & $3.51(1.23)$ & $2.75(0.67)$ & $3.05(0.87)^{\star}$ \\
\hline BI & $2.80(0.41)^{\star \star}$ & $3.18(0.47)^{\star}$ & $2.73(0.38)^{\star}$ & $3.69(1.52)$ & $2.87(0.52)$ & $3.44(0.69)$ \\
\hline \multicolumn{7}{|c|}{$\mathrm{rCBF} / \mathrm{rCBV}:$} \\
\hline Control & $10.7(2.12)$ & $10.1(2.60)$ & $12.1(2.53)$ & $9.98(2.67)$ & $14.4(2.57)$ & $11.7(2.20)$ \\
\hline SBI & $11.7(3.42)$ & $10.8(3.19)$ & $12.5(3.88)$ & $10.3(3.68)$ & $13.8(3.69)$ & $11.5(3.88)$ \\
\hline BI & $10.7(1.57)$ & $9.88(1.83)$ & $11.8(2.40)$ & $9.31(2.88)$ & $12.6(2.48)$ & $9.70(2.29)$ \\
\hline
\end{tabular}

${ }^{\star} \mathrm{p}<0.05 ;{ }^{\star \star} \mathrm{p}<0.01 v$ control group.

$\mathrm{rCBF}=$ regional cerebral blood flow; $\mathrm{rOEF}=$ regional oxygen extraction fraction; $\mathrm{rCMRO}_{2}=$ regional cerebral metabolic rate for oxygen; rCBV=regional cerebral blood volume. 

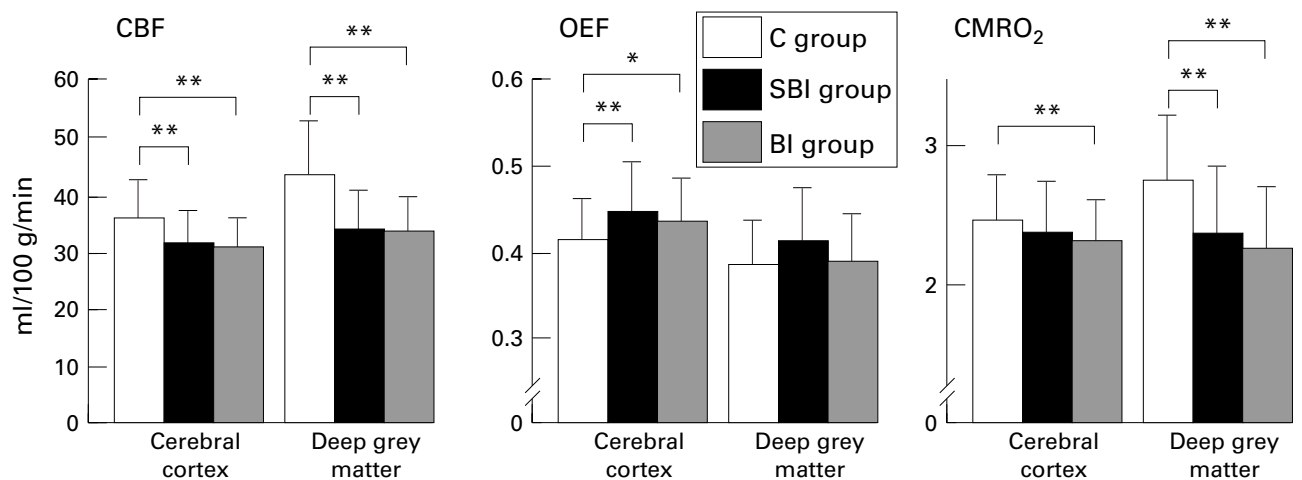

Figure 3 Mean values of $C B F, O E F$, and $C M R O$ of the cerebral cortex and deep grey matter. Mean $C B F$ of the cerebral cortex in the SBI group was lower compared with the control group. Regional CMRO of the cortex in the SBI group was preserved by a compensatory increase in regional OEF. By contrast, mean $C B F$ and $C M R O_{2}$ of deep grey matter in theSBI group were both decreased. $C B F=$ cerebral blood flow; $O E F=$ oxygen extraction fraction; $C M R O_{2}=$ cerebral metabolic rate for oxygen; $C=$ control; $S B I=$ silent brain infarction; $B I=$ brain infarction. ${ }^{\star} p<0.05 ;{ }^{\star \star} p<0.01$.

patients in the BI group tended to have more lesions than those in the SBI group.

PET STUDIES

Table 3 and fig 3 give the values for $\mathrm{rCBF}$, $\mathrm{rOEF}, \mathrm{rCMRO}_{2}, \mathrm{rCBV}$, and $\mathrm{rCBF} / \mathrm{rCBV}$ in the $\mathrm{C}, \mathrm{SBI}$, and BI groups.

Mean values for $\mathrm{CBF}$ in cerebral cortex and deep grey matter were lower in the SBI group (31.6 (SD 5.8) and 34.3 (SD 6.9) $\mathrm{ml} / 100$ $\mathrm{g} / \mathrm{min}$, respectively) and in the BI group (30.8 (5.2), 33.9 (5.9) respectively) than in the C group (36.0 (6.6), 43.5 (9.5) respectively). The mean OEF of the cerebral cortex in both the SBI group and the BI group showed a significant increase compared with the $\mathrm{C}$ group (SBI group 0.45 (0.06), $\mathrm{p}<0.01$ : BI group 0.44 (0.05), p<0.05: C group $0.41(0.05))$. Although the mean $\mathrm{CMRO}_{2}$ of the deep grey matter in the SBI group (2.36 (0.52) $\mathrm{ml} / 100$ $\mathrm{g} / \mathrm{min})$ was significantly decreased $(\mathrm{p}<0.01)$ compared with the $\mathrm{C}$ group (2.76 (0.48), $\mathrm{CMRO}_{2}$ of the cerebral cortex was comparatively preserved (SBI group 2.36 (0.39): C group 2.48 (0.32), fig 3 ).

Regional CBFs of all of the ROIs were significantly smaller in the BI group $(p<0.01-$ 0.05) than in the $C$ group. Regional CBFs of the temporal and parietal cortices, striatum, and thalamus were also significantly lower $(p<0.05)$ in the SBI group than that in the $C$ group. Concentrations of $\mathrm{rCBF}$ in the SBI group fell between the $\mathrm{BI}$ group and the $\mathrm{C}$ group. Regional OEFs in each area were higher in the SBI group than in the $C$ groups, especially in the temporal cortex of the SBI group, which was significantly increased $(\mathrm{p}<0.05)$ compared with the $\mathrm{C}$ group. Regional $\mathrm{CMRO}_{2}$ in the SBI group also fell between the $\mathrm{BI}$ group and the $\mathrm{C}$ group in each area of the brain except for the parietal cortex. Thus $\mathrm{rCMRO}_{2}$ was better preserved in the SBI group compared with the BI group. Regional CBVs of ROIs, except for the occipital cortex and striatum, in the SBI and the BI groups were significantly lower than in the $\mathrm{C}$ group (table 3 ).

\section{Discussion}

The present study shows that mean CBF values for the cerebral cortex and deep grey matter in the SBI group were decreased compared with the $\mathrm{C}$ group. These findings are similar to the recent work by Kobayashi et al, ${ }^{23}$ who have shown that $\mathrm{rCBF}$ measured by xenon-133 inhalation was significantly lower in patients with silent lacunas than in those without lesions. Arteriosclerosis of small cerebral arteries is reported to be closely related to development of SBIs. ${ }^{2-4} 67$ Actually, cerebrovascular resistance presumably due to arteriosclerosis seems to be higher in patients with SBIs than in control subjects. ${ }^{24}$ We speculate that patients with SBIs have advanced cerebral arteriosclerosis and thus CBF measured in the SBI group is lower than that in the $\mathrm{C}$ group. Hence, patients with SBIs have insufficient cerebral circulation. Reduction of $\mathrm{CBF}$ in the SBI group was smaller than in the BI group. Our study also disclosed that the number of lesions in the SBI group tended to be smaller than in the BI group. A previous study showed that patients with SBIs had higher levels of certain risk factors compared with those without stroke, but had lower levels than those with a history of stroke. ${ }^{2}$ Considering these findings, cerebral arteriosclerotic changes may not be as severe in the SBI group compared with the BI group.

It is of interest that changes in CBF and oxygen metabolism in the SBI group seem to differ between the cerebral cortex and deep grey matter. In the present study, both CBF and $\mathrm{CMRO}_{2}$ in the SBI group showed matched hypoperfusion that was significantly smaller than that in the $\mathrm{C}$ group in the striatum and thalamus. By contrast, $\mathrm{CBF}$ in the cerebral cortex was also smaller in the SBI group, but $\mathrm{CMRO}_{2}$ was as well preserved as in the C group. An increase in OEF compensates for the reduction of $\mathrm{CBF}$ to maintain $\mathrm{CMRO}_{2}$. The deep grey matter is perfused by the perforating arteries which are susceptible to the deleterious effects of hypertension. In previous studies, SBI lesions were most often found in these areas. $^{2-4} 24$ Consequently, CBF and oxygen metabolism were more severely diminished in deep grey matter than in the cerebral cortex.

We did not find any significant differences in the ratios of $\mathrm{CBF}$ to $\mathrm{CBV}$ among the three groups. Gibbs et $a l^{25}$ showed that the CBF to CBV ratio was a valuable index of haemodynamic reserve. Meguro et $a l^{26}$ showed that the 
$\mathrm{CBF}$ to CBV ratio of patients with severe periventricular hyperintensity was lower than the moderate to no periventricular hyperintensity groups. They supposed that the CBF to CBV ratio was similar to OEF. By contrast, Powers $e t a l^{7}$ reported that there was no clear relation between the $\mathrm{CBF}$ to $\mathrm{CBV}$ ratio and $\mathrm{OEF}$ and they concluded that the CBF to CBV ratio was neither as sensitive nor as specific for assessing the adequacy of cerebral perfusion relative to cerebral metabolic demands. In the present study, rCBV was significantly smaller in some areas of the brain in both the SBI and $B I$ groups than in the $C$ group. Decreased rCBV and unchanged $\mathrm{CBF}$ to $\mathrm{CBV}$ ratio in patients with BI (either symptomatic or asymptomatic) suggests that there is a baseline narrowing of the vessel lumen, probably due to either advanced arteriolosclerosis or excessive vasoconstriction.

In this study, $\mathrm{rCBF}$ of the striatum and thalamus showed the largest changes, followed by the temporal cortex. Kobayashi et al also disclosed that bilateral frontotemporal regions showed the most remarkable changes. ${ }^{23}$ These areas are supplied by perforating arteries and include many watershed zones. Thus these areas are the most vulnerable regions in both the SBI and the BI groups.

It is a matter for debate how we should treat patients with SBI. Based on the findings in the present study, patients with SBI showed the insufficiency of cerebral circulation compared with control patients. However, to clarify this question, follow up studies of $\mathrm{CBF}$ and metabolism are necessary.

In conclusion, (1) $\mathrm{CBF}$ and metabolism among patients with SBI are impaired compared with control patients. (2) There is matched hypoperfusion in deep grey matter with mild hypoperfusion, but "occult misery perfusion" in the cerebral cortex.

1 Kase CS, Wolf PA,Chodosh EH, et al. Prevalence of silent stroke in patients presenting with initial stroke: the Framingham study. Stroke 1989;20:850-2.

2 Shinkawa A, Ueda K, Kiyohara Y, et al. Silent cerebral infarction in a community-based autopsy series in Japan. The Hisayama study. Stroke 1995;26:380-5.

3 Davis PH, Clarke WR, Bendixen $\mathrm{BH}$, et al, the TOAST investigators. Silent cerebral infarction in patients enrolled in the TOAST study. Neurology 1996;46:942-8.

4 Herderscheê D, Hijdra A, Algra A, et al. Silent stroke in patients with transient ischemic attack or minor ischemic stroke. The Dutch TIA trial study group. Stroke 1992;23. $1220-4$.
5 Jørgensen HS, Nakayama H, Raaschou HO, et al. Silent infarction in acute stroke patients. Prevalence, localization,
risk factors, and clinical significance: the Copenhagen risk factors, and clinical significan
stroke study. Stroke 1994;25:97-104.

6 Laloux P, Ossemann M, Jamart J. Stroke subtypes and risk factors associated with silent infarctions in patients with first-ever ischemic stroke or transient ischemic attack. Acta Neurol Belg 1994;94:17-23.

7 Petersen P, Madsen EB, Brun B, et al. Silent cerebral infarction in chronic atrial fibrillation. Stroke 1987;18:1098-100.

8 Watanabe N, Imai Y, Nagai K, et al. Nocturnal blood presure and silent cerebrovascular lesions in elderly Japanese. Stroke 1996;27:1319-27.

9 Nakamura K, Oita J, Yamaguchi T. Nocturnal blood pressure dip in stroke survivors. A pilot study. Stroke 1995; 26:1373-8.

10 Bornstein NM, Gur AY, Treves TA, et al. Do silent brain infarctions predict the development of dementia after first ischemic stroke? Stroke 1996;27:904-5.

11 Chodosh EH, Foulkes MA, Kase CS, et al. Silent stroke in the NINCDS stroke data bank. Neurology 1988;38:1674-9.

12 EAFT study group. Silent brain infarction in nonrheumatic atrial fibrillation. Neurology 1996;46:159-65.

13 Ricci S, Celani MG, La Rosa F, et al. Silent brain infarctions in patients with first-ever stroke. A community-based study in Umbria, Italy. Stroke 1993;24:647-51.

14 Boon A, Lodder J, Heuts-van Raak L, et al. Silent brain infarcts in 755 consecutive patients with a first-ever supratentorial ischemic stroke. Relationship with index-stroke subtype, vascular risk factors, and mortality. Stroke 1994;25: $2384-90$.

15 Heathfield KWG, Croft PB, Swash M. The syndrome of transient global amnesia. Brain. 1973;96:729-36.

16 Fisher CM. Transient global amnesia-precipitating activities and other observations. Arch Neurol 1982;39:605-8.

17 Caplan L, Chedru F, Lhermitte F, et al. Transient global amnesia and migraine. Neurology. 1981;31:1167-70.

18 Fujii K, Sadoshima S, Ishitsuka T, et al. Regional cerebral blood flow and metabolism in patients with transient global amnesia: a positron emission tomography study. $\mathcal{F}$ Neurol Neurosurg Psychiatry 1989;52:622-30.

19 Zorzon M, Antonutti L, Masè G, et al. Transient global amnesia and transient ischemic attack. Natural history, vascular risk factors, and associated conditions. Stroke 1995;26:1536-42.

20 Frackowiak RSJ, Lenzi GL, Jones T, et al. Quantitative measurement of regional cerebral blood flow and oxygen metabolism in man using ${ }^{15} \mathrm{O}$ and positron emission tomography: theory, procedure, and normal values. $f \mathrm{Com}$ tomography: theory, procedure, and

21 Lammertsma AA, Jones T. Correction for the presence of intravascular oxygen-15 in the steady-state technique for measuring regional oxygen extraction ratio in the brain: 1 . Description of the method. F Cereb Blood Flow Metab 1983; 3:416-24.

22 Fujii K, Sadoshima S, Okada Y, et al. Cerebral blood flow and metabolism in normotensive and hypertensive patients with transient neurologic deficits. Stroke 1990;21:283-90.

23 Kobayashi S, Okada K, Yamashita K. Incidence of silent lacunar lesion in normal adults and its relation to cerebral blood flow and risk factors. Stroke 1991;22:1379-83.

24 Chamorro A, Saiz A, Vila N, et al. Contribution of arterial blood pressure to the clinical expression of lacunar infarction. Stroke 1996;27:388-92.

25 Gibbs JM, Weise RJS, Leenders KL, et al. Evaluation of cerebral perfusion reserve in patients with carotid artery occlusion. Lancet 1984;i:310-4.

26 Meguro K, Hatazawa J, Yamaguchi T, et al. Cerebral circulation and oxygen metabolism associated with subclinical periventricular hyperintensity as shown by magnetic resonance imaging. Ann Neurol 1990;28:378-83.

27 Powers WJ, Raichle ME, Grubb Jr RL. Positron emission tomography to assess cerebral perfusion. Lancet $1985 ; \mathrm{i}$ : $102-3$ 